\title{
Hodgkin's Disease in a Child with Psoriasis
}

\author{
Aziz POLAT ${ }^{1}$, Eda KARADAGLI ${ }^{2}$, Yasemin I. BALCI ${ }^{1}$, M. Levent TASLI ${ }^{3}$, \\ Ali KOCYIGIT ${ }^{4}$, Nilay SENTURK ${ }^{5}$ \\ ${ }^{1}$ Pamukkale University Faculty of Medicine, Department of Pediatric Hematology \\ ${ }^{2}$ Pamukkale University Faculty of Medicine, Department of Pediatrics \\ ${ }^{3}$ Pamukkale University Faculty of Medicine, Department of Dermatology \\ ${ }^{4}$ Pamukkale University Faculty of Medicine, Department of Radyodiology \\ ${ }^{5}$ Pamukkale University Faculty of Medicine, Department of Pathology, Denizli, TURKEY
}

To the Editor,

Psoriasis is a chronic inflammatory disease characterized by erythematous-squamatous plaques with sharp margins. Its prevalence is $1-2 \%$ among the general population, and it constitutes $4 \%$ of the childhood dermatoses. The onset of the disease in the adult is before 20 years of age in about $30 \%$ of the patients (1). Impaired cellular immunity plays an important role in the pathogenesis of psoriasis. Increase in the inflammatory cytokines [tumor necrosis factor- $\alpha$ (TNF- $\alpha)$ ] and interferon- $\gamma($ IFN- $\gamma)$ are observed with the increase in the antigen-presentation related $\mathrm{T}$ Helper-1 cell activation. Inflammatory cytokines have been detected in large amounts in the psoriatic plaques. ${ }^{1}$ Hodgkin's disease (HD) constitutes $40 \%$ of the childhood lymphomas and is observed at a 2-3/100,000 incidence in adolescence. $^{2}$ The etiology of HD has not been clearly understood. However; infectious diseases, genetic tendency, and impaired immunity are known to have roles in development of HD. A permanent disorder is observed particularly in T-cell functioning. It has been believed that the impairment of the cellular immunity is related to the abnormal IL-2 production, as well as increased sensitivity to T-suppressor cells. ${ }^{3}$ In English literature, concomitance of HD and psoriasis has been reported in adults. However, no case of concomitant lymphoma and psoriasis has been reported in childhood previously.

A 16-year-old male with psoriasis had been followed up for 8 years was admitted to our hospital with complaints of puffiness in the neck and supraclavicular region. His physical examination revealed the following: body temperature: $36.7^{\circ} \mathrm{C}$, BP: $110 / 60 \mathrm{~mm} / \mathrm{Hg}$. A painless and immobile lymphadenopathy of $3 \times 1 \mathrm{~cm}$ size was detected in the left anterior cervical chain and a painless, immobile lymphadenopathy of $2 \times 2 \mathrm{~cm}$ size was detected in the left supraclavicular region. No pathological finding was detected except 2-3 psoriatic lesions in the face and left leg. The patient has had received short-term medications of local mometasone furoate cream, sodium fusidate cream, clobetasol propionate hair lotion, calcipotriol solution and salicylic acid coal tar lotion at different intervals due to psoriasis, and PUVA treatment for 3 months. The results of laboratory examinations were as follows: hemoglobin: 12.7 g/dl, hematocrit: $39 \%$, leukocyte count: $12.840 / \mathrm{mm}^{3}$, platelet count: $328.000 / \mathrm{mm}^{3}$, MCV:79fL, MCH:25,2 pg, and MCHC: $31.9 \mathrm{~g} / \mathrm{dL}$. His peripheral blood smear revelaed hypochromic erythrocytes, anisocytosis and poikilocytosis. Atypical blast cell was not observed on his peripheral blood smear. Sedimentation rate was 32 $\mathrm{mm}$ /hour, liver and kidney function tests were normal, and other parameters were as follows; CRP: $1.8 \mathrm{mg} /$ dL, LDH: $173 \mathrm{U} / \mathrm{L}$, uric acid $5.4 \mathrm{mg} / \mathrm{dL}$. Serological tests revealed AntiCMV Ig M and Ig G (-), Ebstein Barr virus Ig M (-), Ig G (+), anti-toxoplasma Ig M(-), Ig G $(-)$, Brucella rose bengal(-), Anti HIV (-).

Mediastinal expansion and suspicious parenchymal nodular appearance within the paracardiac areas were observed in the chest radiography. In the cervical ultrasonography, heterogeneous lymphadenopathies with echogeneous hili and sometimes nodular form were observed in bilateral cervical and jugular chains, the largest one was located in the left supraclavicular region with a $3.5 \mathrm{~cm}$ size. 
The thorax CT evaluation revealed diffuse lymphadenopathies in bilateral supraclavicular regions especially prominent in the left cervical region, anterior and medial mediastinum, and bilateral hilar regions. The left supraclavicular, bilateral mediastinal and hilar lymphadenopathies observed in the thoracal MRI were assessed as nodular consolidation appearances in bilateral pulmonary parenchymes and lymphomatype retention. Abdominal MRI revealed hepatomegaly, and lymph node accumulation in the neighbourhood of splenic hilum, the largest one was $15 \times 9 \mathrm{~mm}$ sized. Positron emission tomography revealed pathologically increased FDG retention was observed in the superior left jugular, left cervical, superior right jugular and right cervical multiple conglomered lymph nodes in the cervical region, supraclavicular, right axillary, inferior jugular, anterior mediastinal, inferior tracheabronchial, subcarinal, left hilar, right hilar, paraoesophageal lymph nodes in the thorax, and hyperdense nodular lesions in bilateral pulmonary parenchyma, and dense FDG retention was observed in a lymph node within the splenic hilum and a mild hypointense focus in inferolateral spleen.

An excisional biopsy examination was performed from the left supraclavicular lymph node. It was observed that the lymph node was divided into nodules with thick sclerotic bands that entirely impairs the normal lymph node structure. Mature lymphocytes, eosinophiles and several plasma cells were observed in the background where Hodgkin-Reed Sternberg (HRS) and its variant neoplastic lymphoid cells were watched. In the immunohistochemical examination, HRS and variant neoplastic lymphoid cells were detected to be CD15 and CD30 (+); CD3 and CD20 (-), the majority of the mature lymphocytes were CD3 (+) T-lymphocyte phenotype, and a minority of them were CD20 (+) B-lymphocyte phenotype. Amyloid was detected in the polarized light microscopy in the hyalinized areas by Congo Red staining, and the diagnosis was made as nodular sclerosing type HD. The cerebrospinal fluid was normal. Hypercellularity and no blast was observed in the bone marrow aspiration smears. Since diffuse lymph node involvement and two extra lymphatic organ involvement (spleen, pulmonary parenchyma), the patient was accepted as having stage 4A Hodgkin's lymphoma and chemotherapy was started for HD.

Psoriasis is a chronic, recurrent, inflammatory, hyperproliferative disease of the skin that has an unknown etiology. In the study of Raychaudri conducted on 707 patients with psoriasis in 2000 , the onset of the disease was before 16 years of age in $31.5 \%$ of the patients. ${ }^{4}$ Our patient has had psoriasis since he was a 8-year-old boy and he received topical treatments according to the progression of the disease at different intervals. The recurrences and remissions were followed-up. In English literature; it has been reported that the risk of Hodgkin's lymphoma has been increased in chronic inflammatory diseases that cause cellular immunity impairment; such as rheumatoid arthritis or Sjögren's syndrome. ${ }^{5}$ The studies reveal that diffuse cellular immunity activation and a cytokine storm related to the increase in the number of $\mathrm{T}$ cells is observed in the pathophysiology of psoriasis (6). Cellular immunity impairment has an important role in the pathogenesis of HD. Therefore, the risk of lymphoproliferative malignancy is increased following psoriasis. ${ }^{7}$

In the study of Gelfand et al. disregarding the drug usage and age factors, the risk of HD was calculated to be increased in similar levels in patients with moderate and severe psoriasis. ${ }^{8}$ The study of Margolis et al. included patients between 20 and 80 years of age, the risk of lymphoproliferative malignancy development among patients with psoriasis who did not received systemic treatment was increased by 2.18 folds. ${ }^{7}$

Systemic biological agents and immunosuppressive drugs used in the treatment of psoriasis include the information that these agents increase the risk of lymphoproliferative malignancy. ${ }^{7}$ In the study of Gelfand et al., patients over 65 years of age were evaluated and the risk of lymphoma was increased by 3 folds. Among the patients with a diagnosis of lymphoma, $1.2 \%$ have methotrexate usage. ${ }^{8}$ Non-Hodgkin's lymphoma was detected in a 46-year old-male patient who received Etenarcept due to psoriasis. ${ }^{9}$ According to the study of Margolis et al., lymphoproliferative disease was determined to be 7.8 folds more common among the patients receiving systemic treatment due to psoriasis who were between 20 and 80 years of age, with the mean follow-up duration of 2.5 year, compared to the same age group of healthy individuals. ${ }^{7}$ It is remarkable that our case was the first for development of HD in the childhood age group without receiving any systemic therapy.

The incidence of HD may be increased in chronic diseases of impaired cellular immunity. The possibility of lymphoma development should be kept in mind 
in chronic diseases with impaired cellular immunity and patients should be closely monitored with this respect.

\section{REFERENCES}

1. Asadullah K, Docke WD, Volk HD, Sterry W. The pathophysiological role of cytokines in psoriasis. Drugs Today 35: 913924, 1999.

2. Eichenauer DA, Borchmann P, Engert A. Adolescents with Hodgkin lymphoma: old children or young adults? Leukemia\&Lymphoma 53: 1257-1262, 2012.

3. Philip Lanzkowsky. Manual of Pediatric Hematology and Oncology. 5th. edition. United Kingdom, Elsevier, 2011: 599-623.

4. Raychaudhuri SP, Gross J. A Comparative study of pediatric onset psorias is with adult onset psoriasis. Pediatr Dermatol 17: 174-178, 2000.

5. Zintzaras E, Voulgarelis M, Moutsopoulos HM. The risk of lymphoma development in autoimmune diseases. Arch Intern Med 165: 2337-2344, 2005.

6. Jeffes III EW, Lee GC, Said S, et al. Elevated numbers of proliferating mononuclear cells in the peripheral blood of psoriatic patients correlate with disease severity. J Invest Dermatol 105: 733-738, 1995.

7. Margolis D, Bilker W, Hennessy S, et al. The risk of malignancy associated with psoriasis. Arch Dermatol 137: 778-783, 2001.

8. Gelfand JM, Berlin J, Van Voorhees A, Margolis DJ. Lymphoma rates are low but increased in patients with psoriasis results from a population-based cohort study in the United Kingdom. Arch Dermatol 139: 1425-1429, 2003.

9. Pai S, Rosenstein ED, Kramer N. Etanercept therapy for psoriatic arthritis in the presence of recurrent non-Hodgkin lymphoma. J Clin Rheumatol 18: 301-303, 2012.

\section{Correspondence}

Dr. Aziz POLAT

Pamukkale Üniversitesi

Tıp Fakültesi Hastanesi

Çocuk Hematolojisi Bilim Dalı, Kınıklı

DENIZLI / TURKEY

Tel: (+90.258) 4440728

e-mail: azizp4@yahoo.com.tr 